\title{
Bilateral polymicrogyria associated with dystonia - a new neurogenetic syndrome?
}

Michal M. Andelman-Gur ${ }^{1}$, Richard J Leventer ${ }^{2,3,4}$, Mohammad Hujirat ${ }^{5}$, Christos Ganos ${ }^{6,7}$, Keren Yosovich ${ }^{8,9,10}$, Nirit Carmi ${ }^{11}$, Dorit Lev ${ }^{1,8,9}$, Andreea Nissenkorn ${ }^{1,12}$, William B.

Dobyns $^{13,14}$, Kailash Bhatia ${ }^{6}$, Tally Lerman-Sagie ${ }^{1,8,12}$, Lubov Blumkin ${ }^{* 1,8,12,15}$

${ }^{1}$ Sackler School of Medicine, Tel-Aviv University, Tel-Aviv, Israel

${ }^{2}$ Department of Neurology, Royal Children's Hospital, Melbourne, Australia

${ }^{3}$ Murdoch Children’s Research Institute, Melbourne, Australia

${ }^{4}$ Department of Pediatrics, University of Melbourne, Melbourne, Australia

${ }^{5}$ Pediatric Neurology Unit, Emek Medical Center, Afula, Israel

${ }^{6}$ Sobell Department of Motor Neuroscience and Movement Disorders, Institute of Neurology, University College London, London, United Kingdom

${ }^{7}$ Department of Neurology, Charité University Hospital Berlin, Berlin, Germany

${ }^{8}$ Metabolic-Neurogenetic Clinic, Wolfson Medical Center, Holon, Israel

${ }^{9}$ Rina Mor Institute of Medical Genetics, Wolfson Medical Center, Holon, Israel

${ }^{10}$ Molecular Genetics Laboratory, Wolfson Medical Center, Holon, Israel

${ }^{11}$ Child Development Center, Maccabi Health Services, Bnei Brak, Israel

${ }^{12}$ Pediatric Neurology Unit, Wolfson Medical Center, Holon, Israel

${ }^{13}$ Departments of Pediatrics and Neurology, University of Washington, Seattle, USA

${ }^{14}$ Center for Integrative Brain Research, Seattle Children's Research Institute, Seattle, USA

${ }^{15}$ Pediatric Movement Disorders Service, Wolfson Medical Center, Holon, Israel

*Correspondence to: Lubov Blumkin MD

Pediatric Neurology Unit, Wolfson Medical Center, Halohamin 62, Holon, Israel Luba.blumkin@gmail.com

Running title: Bilateral polymicrogyria associated with dystonia

This is the author manuscript accepted for publication and has undergone full peer review but has not been through the copyediting, typesetting, pagination and proofreading process, which may lead to differences between this version and the Version of Record. Please cite this article as doi: 10.1002/ajmg.a.61795

This article is protected by copyright. All rights reserved. 


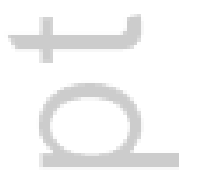

This article is protected by copyright. All rights reserved. 


\begin{abstract}
Background: The clinical presentation of bilateral perisylvian polymicrogyria (PMG) is highly variable, including oromotor dysfunction, epilepsy, intellectual disability and pyramidal signs. Extrapyramidal features are extremely rare.

Methods: We present four apparently unrelated patients with a unique association of PMG with dystonia. The clinical, genetic and radiologic features are described and possible mechanisms of dystonia are discussed.

Results: All patients were female and two were born to consanguineous families. All presented with early childhood onset dystonia. Other neurologic symptoms and signs classically seen in bilateral perisylvian PMG were observed, including oromotor dysfunction and speech abnormalities ranging from dysarthria to anarthria (4/4), pyramidal signs (3/4), hypotonia (3/4), postnatal microcephaly (1/4) and seizures (1/4). Neuroimaging showed a unique pattern of bilateral PMG with an infolded cortex originating primarily from the perisylvian region in three out of four patients. Whole exome sequencing was performed in two out of four patients and did not reveal pathogenic variants in known genes for cortical malformations or movement disorders.
\end{abstract}

Conclusions: The dystonia seen in our patients is not described in bilateral PMG and suggests an underlying mechanism of impaired connectivity within the motor network or compromised cortical inhibition. The association of bilateral PMG with dystonia in our patients may represent a new neurogenetic disorder.

\title{
Key words
}

Polymicrogyria; Malformations of Cortical Development; Magnetic Resonance Imaging; Dystonia 


\section{Introduction}

Polymicrogyria (PMG) is a cortical malformation characterized by overfolding of the cerebral cortex with multiple small gyri and variable lamination abnormalities (Leventer et al., 1999, 2010; Squier and Jansen, 2014). It is one of the most common malformations of cortical development, caused by a disorder in late neuronal migration or in early cortical organization (Barkovich, 2010a). The pathogenesis of PMG is unclear with multiple etiologies implicated, including metabolic disorders, acquired brain injury due to fetal hypoperfusion or intrauterine infection (Barkovich, 2010b), and numerous genetic causes (Guerrini et al., 2008; Jansen and Andermann, 2005; Stutterd et al., 2005/2018). The clinical, pathological and radiological features of PMG are heterogeneous (Leventer et al., 2010, Squier and Jansen, 2014).

Based on the distribution of the cortical malformation, several PMG syndromes have been defined, including bilateral perisylvian, bilateral parasagittal parieto-occipital, bilateral frontal (Barkovich et al., 1999; Guerrini et al., 1997; Guerrini et al., 2000; Kuzniecky et al., 1993; Leventer et al., 2010), asymmetric forms, and unilateral forms (Guerrini and Dobyns, 2014). The most frequent syndrome is bilateral perisylvian PMG that typically presents with epilepsy, intellectual disability, pseudobulbar and pyramidal signs (Brandão-Almeida et al., 2008; Guerrini et al., 2008; Guerrini and Dobyns, 2014; Jansen et al., 2005; Stutterd et al., 2005/2018). Extrapyramidal features have rarely been described (Ganos et al., 2016).

Dystonia is a movement disorder characterized by uncontrolled sustained muscle activation and contractions, generating abnormal postures and movements (Jankovic, 2007; Mink, 2013; Moghimi et al., 2014). Its pathophysiology is not completely elucidated. Dystonia may be associated with dysfunction of one or more components of the sensorimotor network, comprising the basal ganglia, the cerebellar circuitry and cortical areas (Erro et al., 2017; Jinnah and Hess, 2018; Lozeron et al., 2016; Phukan et al., 2011; Standaert, 2011; Tarsy and Simon, 2006). The association of cortical development malformations with dystonia has rarely been described, and was reported in scarce cases of unilateral schizencephaly (Friedman and Stone, 1996; Marinelli et al., 2012) and regional pachygyria (Leuzzi et al., 1993). Dystonia related to bilateral perisylvian PMG has been briefly mentioned in some cases, however it was mainly highlighted in the syndromic association with anarthria/aphonia (Ganos et al., 2016). 
We describe four patients with bilateral PMG who manifested a prominent movement disorder, primarily dystonia. This unique association possibly represents a new neurogenetic syndrome.

\section{Case histories}

\section{Patient 1}

A 7-year-old girl presented to the movement disorder clinic due to severe involuntary movements and global developmental delay. She was a product of a normal full-term pregnancy and delivery, with a birth weight of 2200g (SGA). Unilateral left-sided clubfoot was attributed to a bicornuate uterus. Her parents are healthy second cousins of Israeli Bedouin origin. She has two siblings: her older brother was born with unequal leg length but is otherwise healthy, and her younger sister is healthy. Psychomotor development was delayed: she began sitting at age of 1 year and walked without assistance at 5 years. Speech development was severely impaired, but receptive language and non-verbal communication were perceived as age appropriate based on clinical observation. Bladder and bowel function were normal.

On first neurological examination at age of 7 years, head circumference was $48.5 \mathrm{~cm}$ (2 SD), weight and height were under the fifth percentile (based on Nellhaus growth charts), with no dysmorphic features. She demonstrated generalized rest and action dystonia, most prominent in the neck and the left hand. Antero-lateral-retrocollis in sitting and resting positions was observed. She showed severe difficulties in voluntary facial movements, chewing, swallowing and expressive speech, resulting in persistent open mouth, sardonic smile, drooling and anarthria. Jaw jerk and gag reflexes were normal. She had generalized hypotonia, normal deep tendon reflexes in her upper limbs and right lower limb, and decreased reflexes in the left ankle. No pyramidal signs were found. Independent gait was possible for short distances with severe body torsion. Her fine motor activity was extremely limited by severe dystonia.

On last neurological examination at the age of 12 years, she showed severe generalized dystonia and anarthria. Independent walking had deteriorated and she was wheelchair bound. Non-verbal communication remained age appropriate. She has not undergone a formal 
psychological evaluation. She has received speech, occupational and physical therapy since the age of 2 years, and currently attends a special education school for children with motor disability. Oral baclofen did not improve her symptoms. A combination of trihexyphenidyl and tetrabenazine resulted in partial improvement of dystonia. She has been referred for deep brain stimulation.

Brain MRI showed an extensive bilateral irregular gyral pattern with deep infolding and subtle pebbling of cortical-white matter border, involving the posterior frontal, anterior temporal, perisylvian-parietal, and anterior occipital regions, most severe in perisylvianparietal areas. The deep infolding appeared to originate from the lateral surfaces (not mesial). The white matter in the parietal and temporal lobes was decreased bilaterally, and very broad extended Sylvian fissures were observed [Fig. 1A-D]. The basal ganglia, thalamus, corpus callosum and cerebellum were normal. Metabolic evaluation was normal, including blood electrolytes, liver function, TSH, vitamin B12, lactate, amino acids, very long chain fatty acids and cerebro-spinal fluid analysis (protein, glucose, amino acids and lactate). Muscle biopsy, EEG and chromosome microarray were normal.

Trio whole exome sequencing (using the Agilent V5 kit) was performed in the patient and her parents (see Supplementary Material for details). The analysis did not reveal any pathogenic variants, likely pathogenic or variants of uncertain significance in genes known to be associated with intellectual disability, dystonia, chorea, cortical malformations or brain development. Since the parents were relatives, further analysis for rare homozygous variants was done. This analysis revealed that the patient carries several rare homozygous variants of uncertain significance that segregated with the disease (both parents were heterozygous carriers) in the genes: CAPN8, CLPB, CNIH3, PYGM and USP40. The parents declined further up-to-date exome sequencing.

\section{Patient 2}

A 4-year-old girl presented to the movement disorder clinic due to dystonia and epilepsy. Her parents are healthy second cousins of Israeli Bedouin origin, and she has three healthy siblings. Although her family is from the same tribe as patient 1 , the families are apparently unrelated. 
She was a product of a full-term pregnancy; delivery was by Cesarean section due to breech presentation. Birth weight was 3250g. Gross motor development was delayed: she began sitting at the age of 18 months and walking at the age of 2 years. A left-hand preference was noticed soon after birth. Language development was impaired: single words appeared at the age of 3 years, and currently she speaks in simple phrases.

On examination at the age of 4 years, head circumference was $48.8 \mathrm{~cm}(-1 \mathrm{SD})$, weight and height were normal (50\%ile and 75\%ile, respectively), with no dysmorphic features. Neurological examination demonstrated normal ocular movements, oropharyngeal dysphagia, dysarthria and drooling. She showed mild generalized hypotonia, normal upper limb and patellar tendon reflexes, bilateral non sustained ankle clonus and flexor plantar reflexes. She presented with asymmetric dystonia: action and overflow dystonia of both hands, more prominent on the right; bilateral dystonic posturing of hands; right-sided dystonic hemifacial spasm; dystonic smile and right-sided rest torticollis. She was able to walk, but with a dystonic inner rotation of her right foot, and with dystonic movements of the hands. She spoke in simple phrases but had normal receptive language. Non-verbal communication was normal. Her parents described nocturnal bilateral jerking movements of her hands, that were suspected to be myoclonic seizures.

Brain MRI showed an extensive bilateral irregular gyral pattern with deep infolding of the cortical-white matter border, involving the posterior frontal, anterior temporal, perisylvianparietal, and occipital regions, most severe in the perisylvian-parietal areas. The deep infolding appeared to originate from the lateral surfaces [Fig. 1E-H]. The white matter in the parietal, occipital and temporal lobes was decreased bilaterally. The Sylvian fissures were asymmetric, and the corpus callosum showed abnormal posterior arching. The basal ganglia, thalami and cerebellum were normal. The parents declined genetic investigations. EEG revealed interictal bilateral multifocal epileptic activity, with multiple spikes and generalized spike and wave discharges. She was treated with carbamazepine and levetiracetam for the diagnosis of epilepsy, resulting in partial improvement.

\section{Patient 3}

This article is protected by copyright. All rights reserved. 
A 7-year-old girl presented to the neurology clinic at age nine months due to abnormal movements. She was born at term by Caesarean section for transverse lie following an uncomplicated pregnancy. Her mother was taking thyroxine throughout the pregnancy for Hashimoto's thyroiditis. Birth head circumference was $32 \mathrm{~cm}$ (3\%ile). She is the second child of two healthy unrelated Caucasian parents, and there is no relevant family history, including a healthy elder brother. Her mother had two previous first trimester miscarriages of unknown cause.

Abnormal movements were first noted at age 6 months with dystonic limb posture, fist clenching and tongue thrusting, which occasionally impeded her sucking. A clinical diagnosis of emerging cerebral palsy was made, afterwards occupational therapy and physiotherapy were initiated. Examination by a pediatric neurologist at age 9 months showed no dysmorphic features. Her head circumference was $41.8 \mathrm{~cm}$ (-1.8 SD). She was bright, alert and socially interactive. Eye movements were normal. Tongue thrusting, oro-buccal dyskinesia and dystonic facial movements were noted. She had mild axial hypotonia, head lag, dystonic upper limb posturing and distal limb dyskinesia. Reflexes were 2+ generally with bilateral upgoing plantar responses. Ongoing review showed normal social and receptive language progression, but persistent axial hypotonia, chorea and dystonia and impaired expressive language. Ophthalmology assessment was normal. Between the age of 10 and 18 months her head circumference fell to -3 SD below the mean. Her weight dropped from the $25 \%$ ile to below the 3\%ile and high calorie feeds were introduced.

Benzhexol resulted in modest improvement of her movement disorder. Subsequent trials of trihexyphenidyl, baclofen and diazepam did not significantly improve her movement disorder. Upper limb botulinum toxin injections improved symptoms of painful dystonic posturing. At the age of 7 years she was unable to sit unsupported and had a persistent generalized hyperkinetic movement disorder. She was learning to use an electric wheelchair. She used a handful of single words, but mainly communicated using eye movements, facial expression and head nods, and used a communication book. She was unable to feed herself. There were no concerns regarding her social or cognitive skills, although she had not had formal neuropsychological testing. She was in her second year of mainstream primary school 
and was perceived as excellent at mathematics and spelling. Her head circumference was $47 \mathrm{~cm}$ (-4 SD) and weight $17 \mathrm{~kg}$.

Brain MRI scans were performed at the age of 13 and 26 months. Both studies showed cortical infolding bilaterally in the mid to posterior Sylvian fissures consistent with polymicrogyria [Fig. 1I-L]. The Sylvian fissures were not extended. The basal ganglia were abnormal with small lentiform nuclei and poor demarcation between the globus pallidus and putamen. The rest of the brain appeared normal. Extensive metabolic investigations were normal, including blood electrolytes, liver function, very long chain fatty acids, phytanic acid, TSH, prolactin and vitamin B12; urine pterins; CSF protein, lactate and neurotransmitters, CSF/blood glucose ratio. Chromosome microarray were normal. In 2017, deep sequencing of lymphocyte-derived DNA using a 600X, 330 gene brain malformation panel revealed no variants of significance. Whole exome sequencing targeting genes known to be associated with polymicrogyria was also non-informative. Unfortunately, we could not perform up-to-date genetic analysis since the patient was lost to follow-up.

Patient 4 (presented in brief in Ganos et al., 2016)

A 29-year-old Jamaican lady was evaluated at the neurology clinic due to inability to close her mouth since the age of 2 years. Pregnancy and birth were uneventful and she had normal motor milestones. She never developed expressive language. She did not have swallowing problems though she used her fingers to push food backwards in her mouth so that she could swallow. The patient attended a mainstream school. Previous dental evaluation revealed a malocclusion and open bite anteriorly. Ear nose and throat evaluation showed structurally normal vocal cords. On neurological examination at the age of 29 years, no dysmorphic features were observed; head circumference was within normal range. She had a jaw opening dystonia with dystonic posturing of the left hand. She was able to protrude her tongue but could not elevate it or move it from side to side. She had normal ocular movements; no cranial or peripheral neuropathy, neither cerebellar signs were found. Jaw jerk was brisk and snout reflex was present. She demonstrated deep tendon hyperreflexia. Her gait was spastic. She was treated with botulinum toxin injections and anticholinergics without significant effect.

This article is protected by copyright. All rights reserved. 
Brain MRI showed PMG in both temporal and perirolandic regions extending into the frontal and temporal lobes; absent septum pellucidum and abnormal right olfactory bulb. The corpus callosum was normal [Fig. 1M-P]. The patient declined genetic investigations.

\section{Discussion}

We describe four apparently unrelated patients with bilateral PMG associated with a prominent movement disorder, primarily dystonia. Two of them come from consanguineous families, originating from the same Israeli Bedouin tribe. Three have a unique pattern of infolded PMG with various degrees of severity and cortical areas of involvement. Our patients share similar clinical and radiological features that may represent a new neurogenetic syndrome.

All the patients in our study demonstrated dystonia, which is considered an uncommon manifestation of bilateral PMG. Interestingly, the severity of the movement disorder correlated with the degree of severity of the PMG. The patients presented with additional heterogeneous neurologic symptoms classically seen in bilateral perisylvian PMG: oromotor dysfunction and speech abnormalities ranging from dysarthria to anarthria (4/4), pyramidal signs (3/4), hypotonia (3/4), postnatal microcephaly (1/4) and seizures (1/4). Although no formal neuropsychological assessment was performed, non-verbal cognitive abilities were perceived as normal for age, whereas a significant discrepancy was observed between impaired expressive language and intact receptive language, as expected in bilateral PMG (Braden et al., 2019). Three of the patients demonstrated a unique form of bilateral PMG: a thick cortex, with coarse irregular gyri most prominent in the perisylvian regions, and deep infolding, appearing to originate from the lateral surfaces [Fig. 1A-L, patients 1-3].

Deeply infolded PMG has only recently been defined in the literature. Barkovich (2010b) described the imaging features of 159 PMG patients, and identified six with deep infolding focal PMG. In addition, some of the patients with other types of coarse PMG (mainly perisylvian) were also characterized by deep infoldings of dysmorphic cortex. The clinical features of these patients were not described. Oegema et al. (2019) described subcortical heterotopic gray matter brain malformations (HET), and divided these malformations into five groups. Group 4 included malformations due to abnormal postmigrational development, with 
a subgroup of 19 patients with deeply infolded HET. This subgroup was characterized by extension of two opposing cortices into the deep white matter and mild infolded cortical thickening consistent with PMG, and the patients presented with delayed development, epilepsy and microcephaly, without reported movement disorders. The deeply infolded HET subgroup included a deeply infolded HET-parieto-occipital subtype, which was first described by Guerrini et al. (1997) as "bilateral parasagittal parietooccipital PMG”. No underlying genetic cause was found, and the authors speculated that the etiology of this malformation was acquired rather than genetic. The deeply infolded HET-parieto-occipital subtype shares marked neuroimaging similarities with our three patients, however in our series the infolded cortex was thicker and coarser and originated from the perisylvian regions [Fig1A-L, patients 1-3]. Based on the clinical and radiological unique features of our three patients, compared to the patients described by Oegema et al. (2019), we suggest that our patients have a different disorder, possibly with an underlying genetic mechanism.

The pathogenesis of PMG is still a matter of debate. PMG is associated with a wide range of causative genes. Bilateral perisylvian PMG has been found to be associated with several copy number variants, the most frequent being 22q11.2 deletion and 1p36.3 deletion (Dobyns et al., 2008; Stutterd et al., 2005/2018). In addition, pathogenic variants in tubulin genes (TUBA1A, TUBB, TUBB2A, TUBB2B and TUBB3), in genes involved in microtubule function (DYNC1H1 and KIF5C), mTORopathies (AKT3, PTEN, PIK3CA, PI4KA and PIK3R2) and other pathogenic variants (GRIN1, NDE1, NEEDD4L, PAX6, MAP1B, WDR62 and SCN3A) have been described in association with PMG (Jaglin and Chelly, 2009; Pagnamenta et al., 2015; Smith et al., 2018; Stutterd et al., 2005/2018). To the best of our knowledge, none of these genes have been associated with dystonia or infolded PMG.

Two of our patients underwent genetic studies (whole exome sequencing), which did not reveal any pathogenic variants in known genes associated with cortical malformations or movement disorders. Even though the genetic etiology of the unique association of PMG and dystonia in our patients has not yet been elucidated, we suggest that it originates from a common genetic mechanism, by variants in the same gene or in multiple genes that disrupt common biological pathways (Zohgbi and Warren, 2010). We raise the possibility that our patients share a common neurogenetic syndrome due to the following reasons: the extremely 
unique association between dystonia and PMG observed in our patients; the rare specific radiological features of the PMG (bilateral infolded cortex) observed in three of our patients; the consanguinity in two of the families; and the fact that these two families originate from the same tribe suggesting a possible founder effect. Since two of the families are consanguineous and originate from the same tribe, the inheritance pattern in these families is most likely autosomal recessive. However, in the other two families, other types of inheritance are also possible, such as X-linked dominant, or dominant de novo mutation in genes involving common biological pathways.

The etiology of dystonia is either acquired or genetically determined. The current literature has identified 24 types of hereditary primary dystonia, mostly caused by monogenic mutations (Moghimi et al., 2014); to our knowledge none of these genetic disorders involve PMG. However, there have been rare case studies mentioning dystonic arm posture in patients with unilateral perisylvian PMG (Clark et al., 2010) and spastic-dystonic triparesis in a patient with bilateral perisylvian PMG (Saletti et al., 2007). Other malformations of cortical development have also been described in association with dystonia, such as schizencephaly (Friedman and Stone, 1996; Marinelli et al., 2012) and pachygyria (Leuzzi et al., 1993). Furthermore, Geiger et al. (2017) described a patient with a TUBB2B pathogenic mutation who presented with dystonia associated with pachygyria and dysmorphic basal ganglia.

The underlying mechanism of dystonia in our patients with PMG is not clear. Dystonia has been traditionally associated with dysfunction of the basal ganglia (Standaert, 2011; Tarsy and Simon, 2006). However, recent reports consider dystonia as a network disorder involving not only the basal ganglia, but also the cerebellar pathways (Jinnah and Hess, 2006; Neychev et al., 2008) and various cortical areas, such as the cerebral motor, premotor and sensorimotor cortices (Erro et al., 2017; Lozeron et al., 2016). Hence, dystonia may result from a single-site or multi-site dysfunction within the motor network or from altered connectivity between the components of this network (Carbon and Eidelberg, 2009; Jinnah and Hess, 2018). Three mechanisms have been suggested to underlie the pathophysiology of dystonia: abnormal inhibitory motor circuits, aberrant sensorimotor integration and impaired synaptic plasticity (Quartarone and Hallett, 2013). 
In three of our patients, both the basal ganglia and the cerebellum were anatomically intact whilst patient 3 demonstrated small lentiform nuclei and poor demarcation between the globus pallidus and putamen. This patient also had acquired microcephaly, which was not typical for the rest of our case series. The specific pattern of infolded PMG depicted in three of our patients resulted in a reduced volume of white matter tracts, possibly compromising the motor network. It is possible that the aberrant small gyri impaired white matter tracts in the subcortical circuits, creating impaired connectivity of the basal ganglia with other nodes within the motor network. This theory is consistent with Im et al. (2014) study that found reduced connectivity of white matter tracts in patients with PMG. Interestingly, the severity of the dystonia in our patients was strongly dependent on the severity and distribution of the cortical involvement.

Alternatively, it is possible that the cortical malformation observed in our patients resulted in lack of cortical inhibition, generating the dystonic movements and postures. Hallett (2011) reviewed the neurophysiology of patients with focal hand dystonia, and showed that loss of inhibitory function in the spinal, brainstem and cortical circuits may result in dystonia, possibly due to loss of inhibitory interneurons. Hence, the severe PMG in our patients might have caused a deficit in cortical inhibition, that contributed to the development of the observed dystonia. However, neurophysiological investigations to explore mechanisms of cortical inhibition have not been performed in these patients.

In conclusion: we describe four patients with bilateral PMG presenting with prominent dystonia. The specific pattern of infolded PMG associated with dystonia in three patients (two of them with a consanguineous background) may represent a new neurogenetic disorder.

\section{Declaration of competing interest}

The authors declare that they have no conflict of interest.

\section{Funding}

None.

\section{Author contributions}


M.A.G., R.J.L., C.G., T.L.S. and L.B. collected the data and wrote the article, M.H., K.Y., N.C., D.L., A.N., W.B.D., and K.B. contributed data and critically reviewed the manuscript. 


\section{References}

Barkovich, A.J., Hevner, R., \& Guerrini, R. (1999). Syndromes of bilateral symmetrical polymicrogyria. Am J Neuroradiol, 20, 1814-1821.

Barkovich, A.J. (2010a). Current concepts of polymicrogyria. Neuroradiology, 52, 479-487. doi: 10.1007/s00234-009-0644-2.

Barkovich, A.J. (2010b). MRI analysis of sulcation morphology in polymicrogyria. Epilepsia, 51, 17-22. doi:10.1111/j.1528-1167.2009.02436.x

Braden, R.O., Leventer, R.J., Jansen, A., Scheffer, I.E., \& Morgan, A.T. (2019). Speech and language in bilateral perisylvian polymicrogyria: a systematic review. Dev Med Child Neurol 61, 1145-1152. doi: 10.1111/dmcn.14153.

Brandão-Almeida, I.L., Hage, S.R., Oliveira, E.P., Guimarães, C.A., Teixeira, K.C., Abramides, D.V., Montenegro, M.A., Santos, N.F., Cendes, F., Lopes-Cendes, I., \& Guerreiro, M.M. (2008). Congenital bilateral perisylvian syndrome: familial occurrence, clinical and psycholinguistic aspects correlated with MRI. Neuropediatrics, 39, 139-145. doi: 10.1055/s0028-1085462.

Carbon, M., \& Eidelberg, D. (2008). Abnormal structure-function relationships in hereditary dystonia. Neuroscience, 164, 220-229. doi: 10.1016/j.neuroscience.2008.12.041.

Clark, M., Chong, W.K., Cox, T., \& Neville, B.G. (2010). Congenital perisylvian dysfunction - is it a spectrum? Dev Med Child Neurol, 52, 33-39. doi: 10.1111/j.1469-8749.2009.03348.x. Dobyns, W.B., Mirzaa, G., Christian, S.L., Petras, K., Roseberry, J., Clark, G.D., Curry, C.J., McDonald-McGinn, D., Medne, L., Zackai, E., Parsons, J., Zand, D.J., Hisama, F.M., Walsh, C.A., Leventer, R.J., Martin, C.L., Gajecka, M., \& Shaffer, L.G. (2008). Consistent chromosome abnormalities identify novel polymicrogyria loci in 1p36.3, 2p16.1-p23.1, 4q21.21-q22.1, 6q26-q27, and 21q2. Am J Med Genet A, 146A, 1637-1654. doi: 10.1002/ajmg.a.32293.

Erro, R., Tinazzi, M., Morgante, F., \& Bhatia, K.P. (2017). Non-invasive brain stimulation for dystonia: therapeutic implications. Eur J Neurol, 24, 1228-e64. doi: 10.1111/ene.13363.

Friedman, J.H., \& Stone, W. (1996). Delayed-onset dystonia secondary to unilateral schizencephaly. Mov Disord, 11, 221-222. 
Ganos, C., Crowe, B., Stamelou, M., Kresojević, N., Lukić, M.J., Bras, J., Guerreiro, R., Taiwo, F., Balint, B., Batla, A., Schneider, S.A., Erro, R., Svetel, M., Kostić, V., Kurian, M.A., \& Bhatia, K.P. (2016). The clinical syndrome of dystonia with anarthria/aphonia. Parkinsonism Relat Disord, 24, 20-27. doi: 10.1016/j.parkreldis.2016.01.022.

Geiger, J.T., Schindler, A.B., Blauwendraat, C., Singer, H.S., \& Scholz, S.W. (2017). TUBB2B Mutation in an Adult Patient with Myoclonus-Dystonia. Case Rep Neurol, 9, 216-221. doi: 10.1159/000479788.

Guerrini, R., Barkovich, A.J., Sztriha, L., \& Dobyns, W.B. (2000). Bilateral frontal polymicrogyria: a newly recognized brain malformation syndrome. Neurology, 54, 909-913.

Guerrini, R., Dobyns, W.B., \& Barkovich, A.J. (2008). Abnormal development of the human cerebral cortex: genetics, functional consequences and treatment options. Trends Neurosci, 31, 154-162. doi: 10.1016/j.tins.2007.12.004.

Guerrini, R., \& Dobyns, W.B. (2014). Malformations of cortical development: clinical features and genetic causes. Lancet Neurol, 13, 710-726. doi: 10.1016/S1474-4422(14)70040-7.

Hallett, M. (2011). Neurophysiology of dystonia: The role of inhibition. Neurobiol Dis, 42, 177-184. doi: 10.1016/j.nbd.2010.08.025.

Guerrini, R., Dubeau, F., Dulac, O., Barkovich, A.J., Kuzniecky, R., Fett, C., Jones-Gotman, M., Canapicchi, R., Cross, H., Fish, D., Bonanni, P., Jambaqué, I., \& Andermann, F. (1997). Bilateral parasagittal parietooccipital polymicrogyria and epilepsy. Ann Neurol, 41, 65-73. Im, K., Paldino, M.J., Poduri, A., Sporns, O., \& Grant, P.E. (2014). Altered white matter connectivity and network organization in polymicrogyria revealed by individual gyral topology-based analysis. Neuroimage, 86, 182-193. doi: 10.1016/j.neuroimage.2013.08.011. Jaglin, X.H., \& Chelly, J. (2009). Tubulin-related cortical dysgeneses: microtubule dysfunction underlying neuronal migration defects. Trends Genet, 25, 555-566. doi: 10.1016/j.tig.2009.10.003.

Jankovic, J. (2007). Dystonic disorders. In: Jankovic, J., \& Tolosa, E., editors. Parkinson’s disease and movement disorders.5th ed. Philadelphia, PA: Lippincott Williams and Wilkins, 321-347.

Jansen, A., \& Andermann, E. (2005). Genetics of the polymicrogyria syndromes. J Med Genet, 42, 369-378. doi: 10.1136/jmg.2004.023952. 
Jansen, A.C., Leonard, G., Bastos, A.C., Esposito-Festen, J.E., Tampieri, D., Watkins, K., Andermann, F., \& Andermann, E. (2005). Cognitive functioning in bilateral perisylvian polymicrogyria (BPP): clinical and radiological correlations. Epilepsy Behav, 6, 393-404. doi: 10.1016/j.yebeh.2005.01.012.

Jinnah, H.A., \& Hess, E.J. (2006). A new twist on the anatomy of dystonia: the basal ganglia and the cerebellum? Neurology, 67, 1740-1741. doi: 10.1212/01.wnl.0000246112.19504.61 Jinnah, H.A., \& Hess, E.J. (2018). Evolving concepts in the pathogenesis of dystonia. Parkinsonism Relat Disord, 46 Suppl 1, S62-S65. doi: 10.1016/j.parkreldis.2017.08.001.

Kuzniecky, R., Andermann, F., \& Guerrini, R. (1993). Congenital bilateral perisylvian syndrome: study of 31 patients. The CBPS Multicenter Collaborative Study. Lancet 341, 608612.

Leventer, R.J., Phelan, E.M., Coleman, L.T., Kean, M.J., Jackson, G.D., \& Harvey, A.S. (1999). Clinical and imaging features of cortical malformations in childhood. Neurology, 53, 715-722. doi: 10.1212/WNL.53.4.715.

Leuzzi, V., Ricciotti, V., \& Pelliccia A. (1993). Hemiplegic dystonia associated with regional cortical dysplasia (pachygyria). Mov Disord, 8, 242-244. doi: 10.1002/mds.870080233.

Leventer, R.J., Jansen, A., Pilz, D.T., Stoodley, N., Marini, C., Dubeau, F., Malone, J., Mitchell, L.A., Mandelstam, S., Scheffer, I.E., Berkovic, S.F., Andermann, F., Andermann, E., Guerrini, R., \& Dobyns, W.B. (2010). Clinical and imaging heterogeneity of polymicrogyria: a study of 328 patients. Brain, 133, 1415-27. doi: 10.1093/brain/awq078.

Lozeron, P., Poujois, A., Richard, A., Masmoudi, S., Meppiel, E., Woimant, F., \& Kubis, N. (2016). Contribution of TMS and rTMS in the understanding of the pathophysiology and in the treatment of dystonia. Front Neural Circuits, 10, 90. doi: 10.3389/fncir.2016.00090

Marinelli, L., Bonzano, L., Saitta, L., Trompetto, C., \& Abbruzzese, G. (2012). Continuous involuntary hand movements and schizencephaly: epilepsia partialis continua or dystonia? Neurol Sci, 33, 335-338. doi: 10.1007/s10072-011-0674-5.

Mink, J.W. (2013). Special concerns in defining, studying, and treating dystonia in children. Mov Disord, 28, 921-925. doi: 10.1002/mds.25548. 
Moghimi, N., Jabbari, B., \& Szekely, A.M. (2014). Primary dystonias and genetic disorders with dystonia as clinical feature of the disease. Eur J Paediatr Neurol, 18, 79-105. doi: 10.1016/j.ejpn.2013.05.015.

Neychev, V.K., Fan, X., Mitev, V.I., Hess, E.J., \& Jinnah, H.A. (2008). The basal ganglia and cerebellum interact in the expression of dystonic movement. Brain, 131, 2499-2509. doi: 10.1093/brain/awn168.

Oegema, R., Barkovich, A.J., Mancini, G.M.S., Guerrini, R., \& Dobyns, W.B. (2019). Subcortical heterotopic gray matter brain malformations: Classification study of 107 individuals. Neurology, 93, e1360-e1373. doi: 10.1212/WNL.0000000000008200.

Pagnamenta, A.T., Howard, M.F., Wisniewski, E., Popitsch, N., Knight, S.J., Keays, D.A., Quaghebeur, G., Cox, H., Cox, P., Balla, T., Taylor, J.C., \& Kini, U. (2015). Germline recessive mutations in PI4KA are associated with perisylvian polymicrogyria, cerebellar hypoplasia and arthrogryposis. Hum Mol Genet, 24, 3732-3741. doi: 10.1093/hmg/ddv117.

Phukan, J., Albanese, A., Gasser, T., \& Warner, T. (2011). Primary dystonia and dystonia-plus syndromes: clinical characteristics, diagnosis, and pathogenesis. Lancet Neurol, 10, 10741085. doi: 10.1016/S1474-4422(11)70232-0.

Quartarone, A., \& Hallett, M. (2013). Emerging concepts in the physiological basis of dystonia. Mov Disord, 28, 958-967. doi: 10.1002/mds.25532.

Saletti, V., Bulgheroni, S., D'Incerti, L., Franceschetti, S., Molteni, B., Airaghi, G., Pantaleoni, C., D'Arrigo, S., \& Riva, D. (2007). Verbal and gestural communication in children with bilateral perisylvian polymicrogyria. J Child Neurol, 22, 1090-1098.

Smith, R.S., Kenny, C.J., Ganesh, V., Jang, A., Borges-Monroy, R., Partlow, J.N., Hill, R.S., Shin, T., Chen, A.Y., Doan, R.N., Anttonen, A.K., Ignatius, J., Medne, L., Bönnemann, C.G., Hecht, J.L., Salonen, O., Barkovich, A.J., Poduri, A., Wilke, M., de Wit, M.C.Y., Mancini, G.M.S., Sztriha, L., Im, K., Amrom, D., Andermann, E., Paetau, R., Lehesjoki, A.E., Walsh, C.A., \& Lehtinen, M.K. (2018). Sodium Channel SCN3A (Na(V)1.3) Regulation of Human Cerebral Cortical Folding and Oral Motor Development. Neuron 99, 905-913.e7. doi:10.1016/j.neuron.2018.07.052.

Squier, W., \& Jansen, A. (2014). Polymicrogyria: pathology, fetal origins and mechanisms. Acta Neuropathol Commun, 2, 80. doi: 10.1186/s40478-014-0080-3. 
Standaert, D.G. (2011). Update on the pathology of dystonia. Neurobiol Dis, 42, 148-151. doi: 10.1016/j.nbd.2011.01.012.

Stutterd, C.A., Dobyns, W.B., Jansen, A., Mirzaa, G., \& Leventer, R.J. (2005, Updated 2018). Polymicrogyria overview. In: Adam, M.P., Ardinger, H.H., Pagon, R.A., et al., editors. (19932019). GeneReviews ${ }^{\circledR}$ [Internet]. Seattle (WA): University of Washington, Seattle. Available from: https://www.ncbi.nlm.nih.gov/sites/books/NBK1329/

Tarsy, D., \& Simon, D.K. Dystonia. (2006). $N$ Engl J Med, 355, 818-829. doi: 10.1056/NEJMra055549

Zoghbi, H.Y., Warren, S.T. (2010). Neurogenetics: advancing the "next-generation" of brain research. Neuron 68:165-73. 


\section{Figures}

Figure 1: Polymicrogyria of the patients in our study

Neuroimaging showing bilateral perisylvian PMG in our patients, each column depicts images from the same patient: patient 1 A-D, patient $2 \mathrm{E}-\mathrm{H}$, patient $3 \mathrm{I}-\mathrm{L}$, patient $4 \mathrm{M}-\mathrm{P}$.

Axial T2-weighted (A-B, D) and T1-weighted (C) MR imaging: an extensive bilateral irregular gyral pattern with deep infolding in the posterior frontal (B, white arrows), anterior temporal (D), perisylvian-parietal (C, D), and anterior occipital regions (A, C), most severe in perisylvian-parietal areas. The deep infolding appears to originate from lateral surfaces (B, white arrows). The white matter in the parietal and temporal lobes is decreased bilaterally (B, C, arrow heads). Sylvian fissures are broadly extended (D).

Axial T2-weighted (F), Fluid-attenuated inversion recovery-FLAIR (G) and T1-weighted (E, H) MR imaging: deep infolding in the posterior frontal, anterior temporal, perisylvian-parietal, and occipital regions, most severe in perisylvian-parietal areas (F, G). The Sylvian fissures are asymmetric (E, F, G).

Axial T2-weighted (I, K) and T1-weighted (J, L) MR imaging: cortical infolding bilaterally in the mid to posterior Sylvian fissures consistent with polymicrogyria, right more than left (I-L). The Sylvian fissures are not extended (K, L). Abnormal basal ganglia with small lentiform nuclei and poor demarcation between the globus pallidus and putamen (L).

Axial T2-weighted MR imaging (M-P): cortical overfolding in the temporal and perirolandic regions bilaterally extending into the frontal and temporal lobes consistent with polymicrogyria (white arrows). 


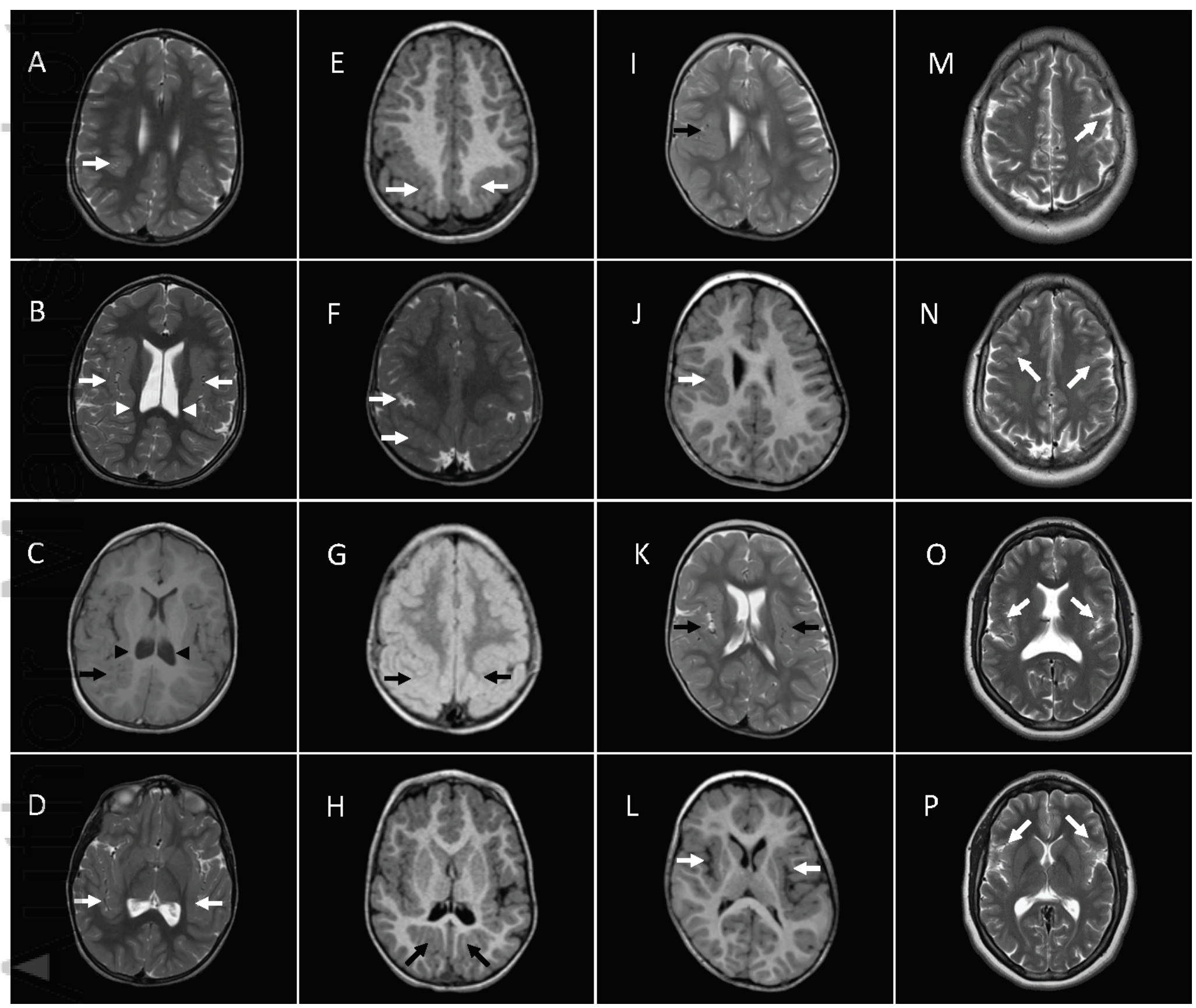

AJMGA_61795_Figure_PMG_for_sub.tiff 


\section{University Library}

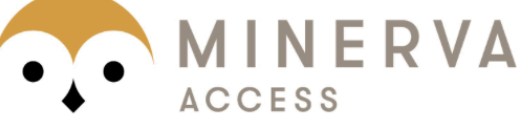

A gateway to Melbourne's research publications

Minerva Access is the Institutional Repository of The University of Melbourne

Author/s:

Andelman-Gur, MM;Leventer, RJ;Hujirat, M;Ganos, C;Yosovich, K;Carmi, N;Lev,

D;Nissenkorn, A;Dobyns, WB;Bhatia, K;Lerman-Sagie, T;Blumkin, L

Title:

Bilateral polymicrogyria associated with dystonia: A new neurogenetic syndrome?

Date:

2020-08-17

Citation:

Andelman-Gur, M. M., Leventer, R. J., Hujirat, M., Ganos, C., Yosovich, K., Carmi, N., Lev, D., Nissenkorn, A., Dobyns, W. B., Bhatia, K., Lerman-Sagie, T. \& Blumkin, L. (2020). Bilateral polymicrogyria associated with dystonia: A new neurogenetic syndrome?.

AMERICAN JOURNAL OF MEDICAL GENETICS PART A, 182 (10), pp.2207-2213. https:// doi.org/10.1002/ajmg.a.61795.

Persistent Link:

http://hdl.handle.net/11343/276152 